\title{
3-D Microwave Imaging for Breast Cancer
}

\author{
George CHENG, Yong ZHU, Jan GRZESIK \\ Allwave Corporation, S3860 Del Amo Boulevard, Suite 404, Torrance, CA 90503, USA \\ george@allwavecorp.com, yong@allwavecorp.com, jan@allwavecorp.com
}

\begin{abstract}
We introduce a novel microwave imaging technique for breast cancer detection. Our approach provides a one-pass inverse image solution, which is completely new and unprecedented, unrelated to tomography or radarbased algorithms, and unburdened by the optimization toil which lies at the heart of numerical schemes. It operates effectively at a single frequency, without requiring the bandwidth of radar techniques. Underlying this new method is our unique Field Mapping Algorithm (FMA), which transforms electromagnetic fields acquired upon one surface, be it through outright measurement or some auxiliary computation, into those upon another in an exact sense.
\end{abstract}

\section{Keywords}

Electromagnetic waves, imaging, inverse scattering.

\section{Introduction}

We propose herein a diagnostic method based upon the Field Mapping Algorithm (FMA), which we have developed to provide closed-form solutions to the problems associated with 3-D microwave holography. The FMA transforms electromagnetic fields upon one surface into those upon another in an exact sense [1]-[9]. It determines the entire electric and magnetic field everywhere based on two tangential field components, either electric or magnetic, on the data acquisition surface, be it a plane, a sphere, or a cylinder.

The FMA was first derived in 2004 for use in radar imaging based on microwave holography. We viewed the imaging objective as an inverse source problem, and solved the underlying integral equations exactly. In this way we have found rigorous, closed-form solutions to electromagnetic holography problems utilizing planar, spherical, and cylindrical near-field data. As a direct result of our FMA, all components of both electric and magnetic fields interior to the source region can be determined exactly based on two-component tangential electric or magnetic field information collected on exterior enclosures of rectangular, spherical, or cylindrical type. Furthermore, despite its initial, free-space use in the context of radar, it remains valid when applied to inhomogeneous media.
In our derivation of the FMA we began with the basic wave equation

$$
\nabla \times \nabla \times \mathbf{E}(\mathbf{r})-k^{2} \mathbf{E}(\mathbf{r})=0
$$

for $\mathbf{r}$ in the source-free region, with the boundary condition $\mathbf{E}^{\mathrm{t}}(\mathbf{r})=\mathbf{E}_{\mathrm{s}}^{\mathrm{t}}(\mathbf{r})$ when $\mathbf{r}$ lies on the surface $S_{i}, i=1,2,3$ respectively for rectangular, spherical, and cylindrical enclosing surfaces, and where $\mathbf{E}_{\mathrm{s}}{ }^{\mathrm{t}}(\mathbf{r})$ stands for the two tangential components of the electric field over the enclosure. We then derived exact solutions in closed form for the field interior to the surface (the lengthy mathematical manipulations are compiled in [9]) as follows:

$$
\begin{aligned}
& \mathbf{E}(\mathbf{r})=\iint \mathbf{E}_{\mathrm{s}}^{\mathrm{t}}\left(\mathbf{r}_{\mathbf{s}}\right) \mathbf{P}_{i}\left(\mathbf{r}, \mathbf{r}_{\mathbf{s}}\right) \mathrm{d} S, i=1,2,3 \\
& \mathbf{H}(\mathbf{r})=\iint \mathbf{E}_{\mathrm{s}}^{\mathrm{t}}\left(\mathbf{r}_{\mathbf{s}}\right) \mathbf{Q}_{i}\left(\mathbf{r}, \mathbf{r}_{\mathbf{s}}\right) \mathrm{d} S, i=1,2,3
\end{aligned}
$$

where $\mathbf{E}(\mathbf{r})$ and $\mathbf{H}(\mathbf{r})$ comprise together all six components of the electric and magnetic field interior to the enclosure S. $\mathbf{E}_{\mathrm{s}}{ }^{\mathrm{t}}\left(\mathbf{r}_{\mathrm{s}}\right)$ embodies the two tangential electric field components over the surface, which are known from a priori computation or else measurement, while $\mathbf{P}_{i}\left(\mathbf{r}, \mathbf{r}_{\mathbf{s}}\right), \mathbf{Q}_{i}\left(\mathbf{r}, \mathbf{r}_{\mathbf{s}}\right)$ are closed-form kernels respectively for the electric and magnetic fields. It should be noted that $\mathbf{E}_{\mathrm{s}}^{\mathrm{t}}\left(\mathbf{r}_{\mathrm{s}}\right), \mathbf{P}_{i}\left(\mathbf{r}, \mathbf{r}_{\mathrm{s}}\right)$, and $\mathbf{Q}_{i}\left(\mathbf{r}, \mathbf{r}_{\mathbf{s}}\right)$ in (2) and (3) are all given in matrix format, and that electric surface data $\mathbf{E}_{\mathrm{s}}{ }^{\mathrm{t}}\left(\mathbf{r}_{\mathrm{s}}\right)$ can be replaced with magnetic surface data $\mathbf{H}_{\mathrm{s}}^{\mathrm{t}}\left(\mathbf{r}_{\mathrm{s}}\right)$, provided that both $\mathbf{P}_{i}\left(\mathbf{r}, \mathbf{r}_{\mathbf{s}}\right)$ and $\mathbf{Q}_{i}\left(\mathbf{r}, \mathbf{r}_{\mathbf{s}}\right)$ are suitably modified.

In its application to breast cancer detection, our system consists of a single RF transmit source (either a horn or a dish) on one side of the organ, and an array of receive sensors at the other end, with the breast target situated in between. The required $3 \mathrm{D}$ imaging of breast tissue is reconstructed from the data thus collected as a direct FMA transform. It should be stressed that, unlike the procedures followed in conventional microwave imaging, with its multitude of data acquisition planes surrounding the target organ, the data collection here is confined to just one gathering plane, at a single frequency. The entire process, including measurement and data processing, would typically consume just a few minutes.

The internal geometry of the mammary gland and, in particular, any suspect pathological inclusions, are then ascertained from the field polarization attributes, which correlate with tissue structure. In particular, the tissue mass 
associated with any given field distribution can thus be revealed as to its overall configuration and electrical properties by way of the field polarization attributes.

Our suggested diagnostic procedure does not inflict the discomfort and potential injury accompanying breast compression, which is an essential part of the standard mammography regimen, nor does it insists upon the untidy aspects of fluid immersion as required in conventional microwave imaging.

We substantiate the power of our FMA diagnostic procedure with two measurement test cases involving actual biological tissues. These results are full of promise as to the utility of microwaves as a non-invasive diagnostic tool.

\section{One-pass Medical Diagnosis Using Microwaves}

Fig. 1 conveys the general arrangement of our onepass microwave diagnosis procedure utilizing a single transmitter and a multiple array pickup, with both source (Tx) and measurement (Rx) equipment placed at some remove from the patient's torso, the actual distances being set through a discretionary choice of convenience for both patient and attending clinician. The information gathered at array $\mathrm{Rx}$ is then processed under the control of the FMA so as to yield a full volumetric map of the patient's internal structure, providing thus a non-invasive disclosure of any pathology which may have crept in.

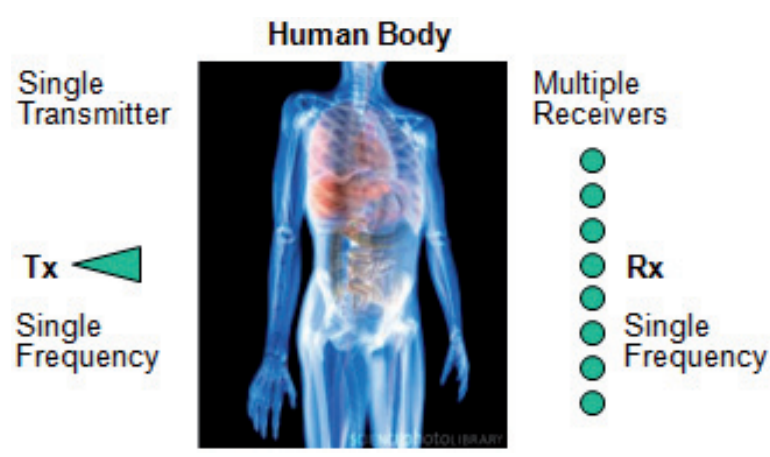

Fig. 1. Human body, with single Tx and multi-Rx microwave transmission.

In the specific context of breast cancer detection, an application raised to paramount urgency by the prevalence of this malady, the microwave Tx-Rx-FMA diagnosis trio may then be incorporated into clinical practice after the schematic fashion suggested in Fig. 2. It is especially to be noted in Fig. 2 that the prospective patient is subjected neither to the pain of breast compression, nor to the discomfort of mammary immersion in fluid, two of the many defects which afflict existing procedures for breast cancer diagnosis, the first in connection with the established practice of x-ray mammography, and the second with the emerging, still largely research-level efforts utilizing a multitude of microwave elements in both $\mathrm{Rx}$ and $\mathrm{Tx}$ modes, followed by malignancy localization on the basis of numerical optimization requiring massive amounts of computer time [10]-[13].

\section{Application: Breast Cancer Detection}

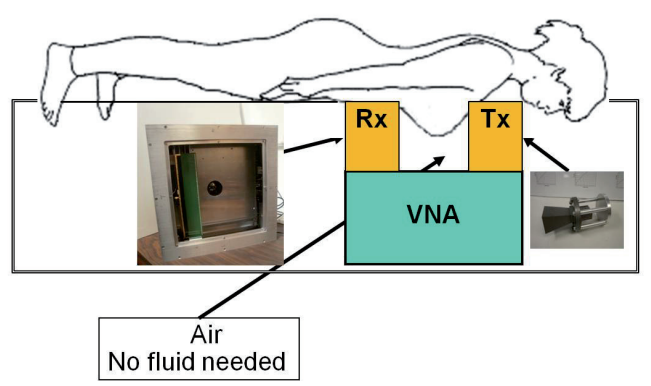

Fig. 2. Three-pronged Tx-Rx-FMA breast cancer detection.

\section{Internal Structure Detection in Biological Tissue Surrogates}

As a preliminary to any sort of clinical testing with human subjects, we have utilized pork meat samples, both with bone (Case 2) and without (Case 1), as suitable surrogate targets for the type of microwave probing which is organized beneath our Tx-Rx-FMA triad. Near-field microwave data were acquired in a standard anechoic chamber as is shown in Fig. 3, using a y-directed array source on the left and a moveable waveguide detection probe on the right.
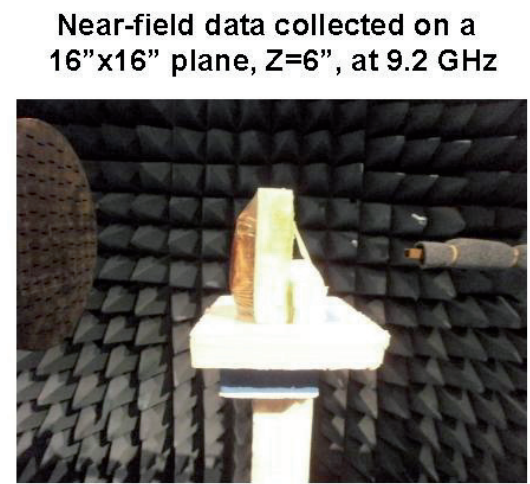

Fig. 3. Near-field microwave measurement in chamber with $\mathrm{y}$-directed array Tx and movable waveguide Rx.

\subsection{Case 1: Boneless Pork Sample}

In Fig. 4 we show near-field, $9.2 \mathrm{GHz}$ microwave data following its transmission across a boneless pork sample and gathered across a 16"x16" frame 6" downstream. Pork tissue reconstructed across 12"x12" cross sectional slices under the control of our FMA appears in Fig. 5, which offers convincing testimony to the absence of internal bone structure. Fig. 6 provides a more dramatic, comprehensive overview of all such slices from the vantage point of various angles. 


\section{Pork Sample under Test}

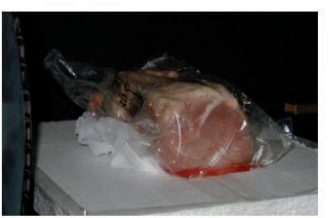

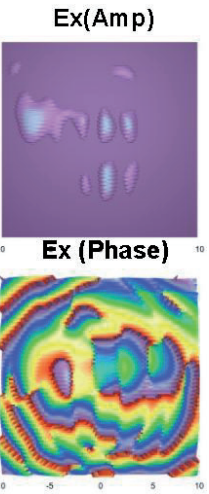

Fig. 4. Measured microwave data following transmission across a pork sample with bone.
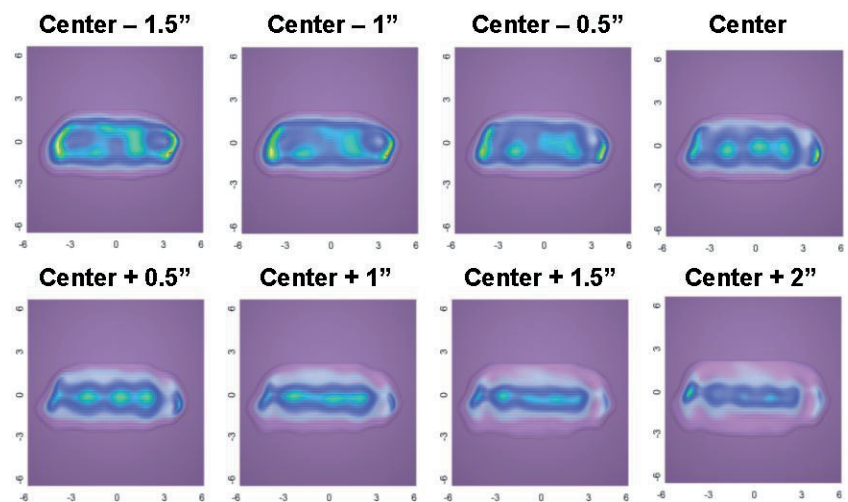

Fig. 5. Boneless pork tissue reconstructed under FMA control.

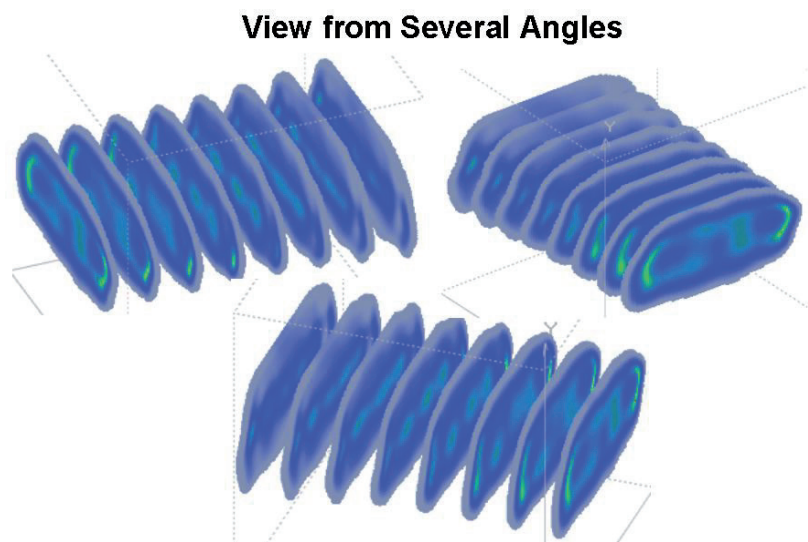

Fig. 6. Boneless FMA slice views from various angles.
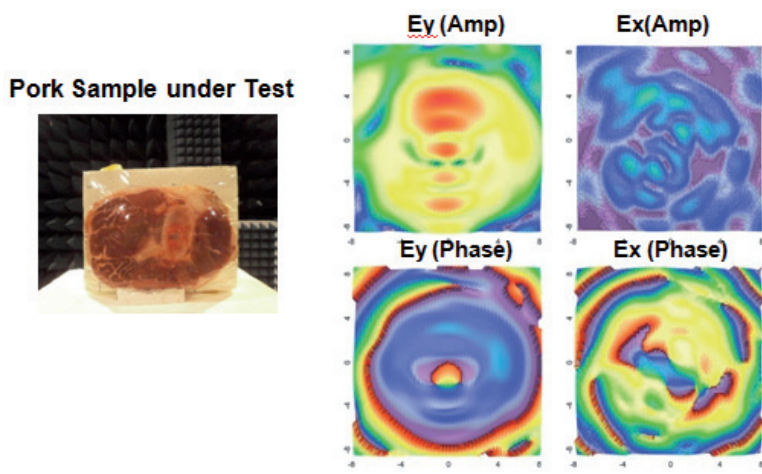

Fig. 7. Measured microwave data following transmission across a pork sample with bone.

\subsection{Case 2: Pork with Bone}

With bones present, Fig. 7 and 8 become the respective counterparts of Fig. 4 and 5. Fig. 7, this time across merely 8"x8" cross-sectional slices but still at $9.2 \mathrm{GHz}$, shows quite clearly the power of our FMA to reveal bone structure.
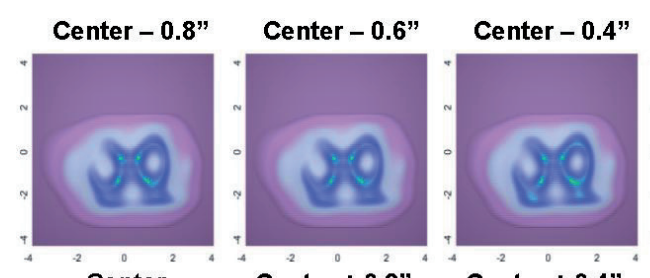

Center - 0.2"
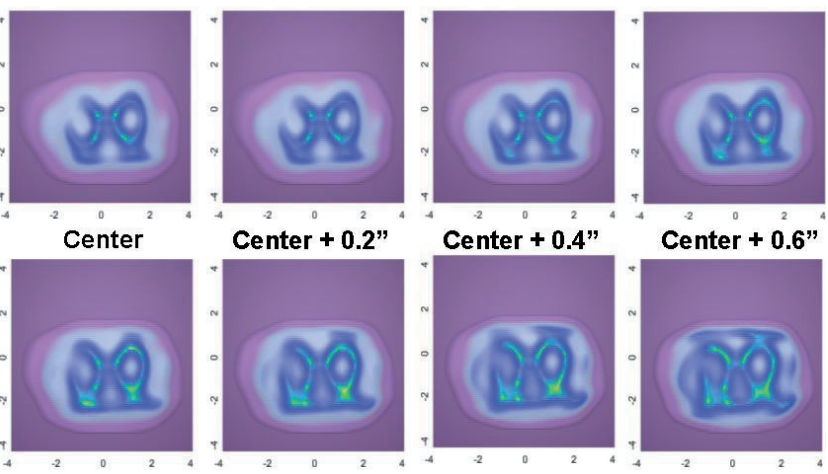

Center + 0.6"

Fig. 8. Pork tissue with bone reconstructed FMA control.

\subsection{Case 3: Allwave Portable Array Scanner: Boneless Beef}

In addition to the anechoic chamber data collection method illustrated in Fig. 3, Allwave has developed a portable microwave scanner with a genuine linear array $\mathrm{Rx}$ pickup, shown in Fig. 9 with boneless beef tissue under test.

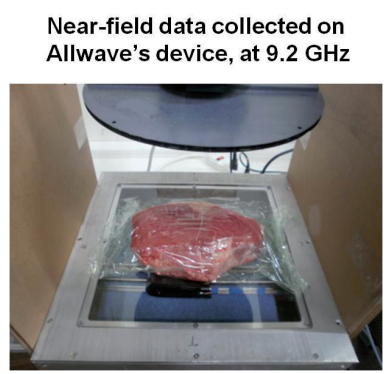

Fig. 9. Boneless beef tissue probed on Allwave's portable scanner.
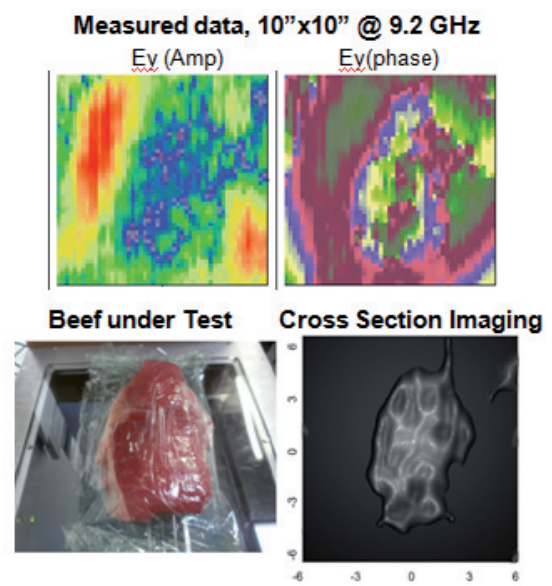

Fig. 10. Boneless beef tissue: measured data and FMA reconstruction. 
Fig. 10 summarizes the (boneless) beef tissue setup, with measured microwave data, acquired across a 10"x10" frame in both amplitude and phase, in the upper row, and an FMA reconstruction through the center at lower right. The structural fidelity of that reconstruction is compelling without any need for additional comment.

\section{FMA Field Reconstruction with Non-biological Targets}

While biological specimens, and, in particular, cancer detection in breast tissue, define our primary arena of concern, it is of great value to witness the power and versatility of FMA field reconstruction vis-à-vis inanimate targets. With this objective in mind, we consider the following examples:

A) slotted sphere with thin azimuthal voltage gap: spherical near-field data is computed on the basis of an eigenfunction expansion across an enclosing sphere, and the FMA then reconstructs field on inner source sphere (Fig. 11);

B) measured near-field of patch antenna with blockage at $2.4 \mathrm{GHz}$ : data capture on 60"x60" plane aperture 18 " downstream allows full volume FMA reconstruction upstream, with diffractive phenomena around the blockage visible as expected (Fig. 12);

C) shape of a model airplane is recovered on the basis of its measured near field at $9.2 \mathrm{GHz}$ when processed under FMA control, providing in effect a single-frequency ISAR image (Fig. 13); and

D) metallic objects concealed within a multilayered dielectric material are revealed at their various depths following FMA processing of near-field planar data acquired $5 "$ from the material at 9.2 GHz (Fig. 14, 15).

A. Slotted sphere with thin voltage gap: Input data analytically generated

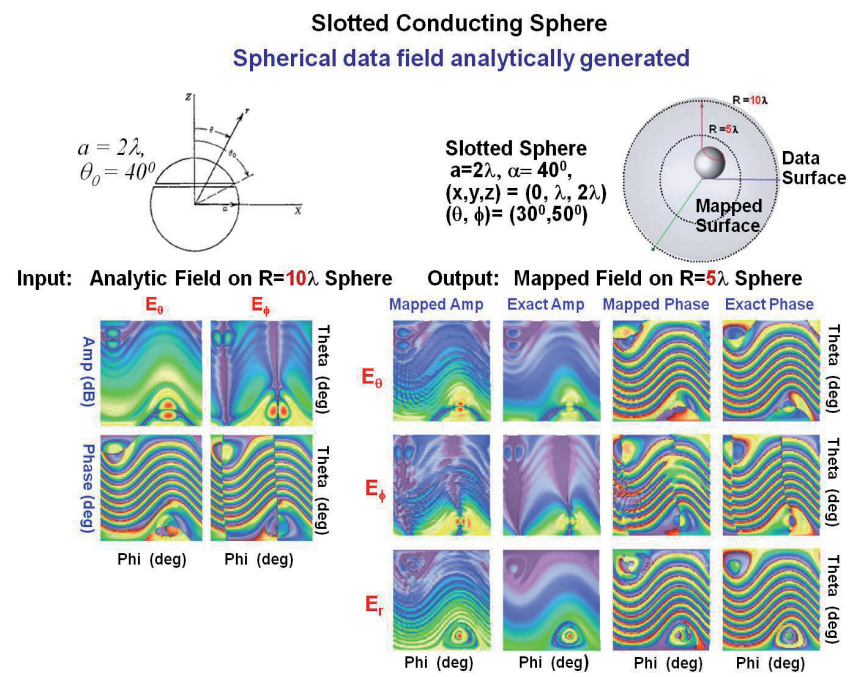

Fig. 11. Slotted sphere with thin voltage gap: field data on input sphere analytically generated.
B. Patch Antenna with Blockage:

Measured/Reconstructed Near Field at $2.4 \mathrm{GHz}$

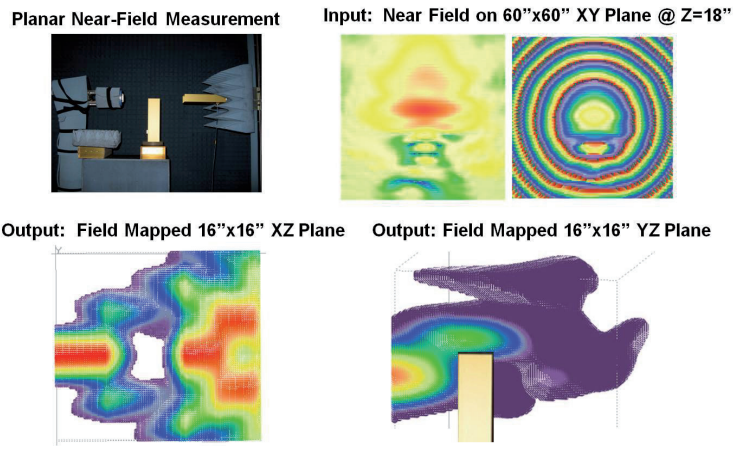

Fig. 12. Patch antenna with blockage: measured planar near field 8 " away from the blockage at $2.4 \mathrm{GHz}$, as input data.

C. Aircraft Model Shape Measurement/Reconstruction in Near Field at $9.2 \mathrm{GHz}$

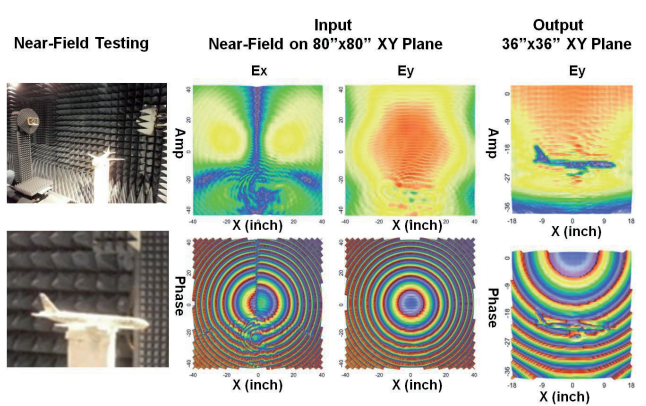

Fig. 13. Aircraft model shape measurement/reconstruction in near field at $9.2 \mathrm{GHz}$.

D. Metallic Obstacle Detection within Multilayered Dielectric Material at $9.2 \mathrm{GHz}$

Multilayered Material

Measurement Setup Input: Measured Data at Z=5", $9.2 \mathrm{GHz}$
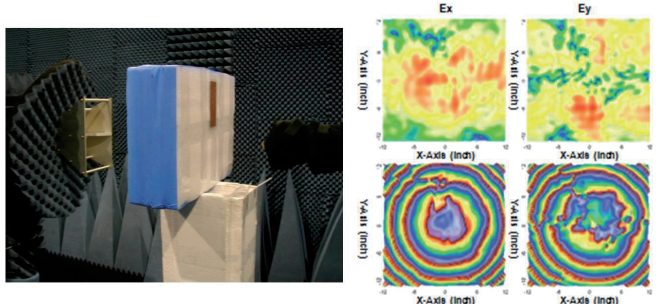

Fig. 14. Chamber measurement of near field data following transit across metallic obstacles embedded in dielectric layers $(9.2 \mathrm{GHz})$.

Output: Mapped Field @ 9.2 GHz

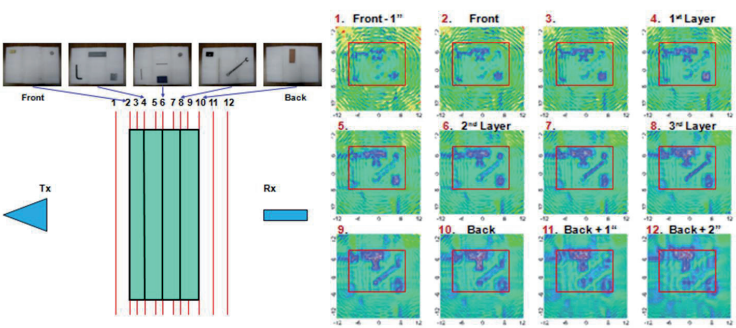

Fig. 15. Metallic obstacles reconstruction at various depths on the basis of FMA processing with the near-field data from Fig.14 as input. 


\section{Conclusion}

The diagnostic/reconstructive power of our FMA algorithm is quite evident in imaging both biological tissues in cases 1-3, and all four inanimate targets in cases A-D. In cases 1-3, the imaging of tissue in various cross sections reveals their respective structures as boneless, boned, and visible tendon. Moreover, cases A-D provide excellent validating examples. Particularly noticeable in case $\mathrm{C}$ is the ability conferred by FMA to image an aircraft using just a single frequency, a task which normally requires widespectrum data in conventional radar techniques. Repeated success of this sort offers great hope and incentive that application of our FMA, especially in medicine, will shortly provide a potent weapon against the scourge of breast cancer and other afflictions weighing upon mankind.

\section{References}

[1] CHENG, G. G., ZHU, Y., GRZESIK, J. Imaging by near-field measurements. In AMTA 2009 Proceedings. Salt Lake City (USA), 2009, A09-0111.

[2] CHENG, G. G., ZHU, Y., GRZESIK, J. Exact solutions for microwave holography - Part I: Planar case. In PIERS 2009 Proceedings. Beijing (China), 2009.

[3] CHENG, G. G., ZHU, Y., GRZESIK, J. Exact solutions for microwave holography - Part II: Cylindrical and spherical cases. In PIERS 2009 Proceedings. Beijing (China), 2009.

[4] CHENG, G. G., ZHU, Y., GRZESIK, J. Visualization of electromagnetic waves. In AMTA 2008 Proceedings. Boston (USA), 2008, A08-0061.

[5] CHENG, G. G., ZHU, Y., GRZESIK, J. Microwave photography. In IEEE APS 2008 International Symposium Digest. San Diego (USA), 2008, IF219.10.

[6] CHENG, G. G., ZHU, Y., GRZESIK, J. Full space wave construction via single near-field measurement. In AMTA 2007 Proceedings. St. Louis (USA), 2008, A07-0018.

[7] CHENG, G. G., ZHU, Y., GRZESIK, J. Wave diffraction phenomenon via measurement. In EuCAP 2007 Proceedings. Edinburgh (UK), 2007, Th2.11.10.

[8] CHENG, G. G., ZHU, Y., HUYNH, S., CHANG, F. C. RCS time domain near field measurement and 3D ISAR. In AMTA 2004 Proceedings. Atlanta (USA), 2007, PID-207.

[9] CHENG, G. G. Field mapping algorithm. Allwave Corporation Technical Report, No. AW015-32. 2004.
[10] IRISHINA, N. Microwave medical imaging using level set techniques. Ph.D. Thesis. Universidad Carlos III de Madrid, Spain, 2004.

[11] FEAR, E. C., LI, X., HAGNESS, S., STUCHLY, M. Confocal microwave imaging for breast cancer detection: Localization of tumors in three dimensions. IEEE Transactions on Biomedical Engineering, August 2002, vol. 49, no. 8, p. 812-822.

[12] BOND, E. J., LI, X., HAGNESS, S., VEEN VAN, B. Microwave imaging via space-time beamforming for early detection of breast cancer. IEEE Transactions on Antennas and Propagation, August 2003, vol. 51, no. 8, p. 1690-1705.

[13] FEAR, E. C., HAGNESS, S., MEANEY, P., OKONIEWSKI, M., STUCHLY, M. Enhancing breast tumor detection with near- field imaging. IEEE Microwave Magazine, March 2002, vol. 3, p. 48 to 56.

\section{About the Authors}

George G. CHENG was born in Taiwan in 1948. He received his Ph.D. from the University of California at Berkeley in 1984. His professional interests in the aerospace industry throughout three decades include satellite communications, antenna systems, RCS, imaging, inverse scattering, and RF test systems. He is currently with Allwave Corporation and is involved in projects in the areas of microwave medical imaging, materials measurement techniques, and array sensor detection systems.

Yong ZHU was born in Hangzhou, China, in 1971. He received his Ph.D. degree in Industrial Automation from Zhejiang University, Zhejiang, China in 1998. His research interests include microwave imaging, AI, signal processing and wireless communications. Currently he is with Allwave Corporation and is involved in a project of microwave medical imaging.

Jan A. GRZESIK was born in Rybnik, Poland, in 1939. He received his B. A. in Physics from UCLA in 1960 (highest honors), his M. A. in Physics from Harvard University in 1961, and his Ph.D. in Nuclear Engineering from UCLA in 1977. He has worked in a variety of contexts in applied science (hydrodynamic impact, radiant heat transport, neutron transport, neutron thermalization, ion orbit computation, ion cyclotron resonance isotope separation, Monte Carlo simulation, etc.), with a continuing emphasis throughout on antennas and classical electrodynamics. 